\title{
Timing and Carrier-Envelope Phase Properties of Optical Parametric Generation driven by Femtosecond Pulses
}

\author{
C. Manzoni \\ Center for free Electron Laser Science, Max Planck Research Group for Structural Dynamics \\ c/o DESY, Notkestraße, 85 - 22607 Hamburg, Germany \\ cristian.manzoni@mpsd.cfel.de \\ G. Cirmi \\ Massachusetts Institute of Technology \\ 77, Massachusetts Avenue, Cambridge MA-02139 USA \\ D. Brida, S. De Silvestri and G. Cerullo \\ National Laboratory for Ultrafast and Ultraintense Optical Science - INFM-CNR, \\ Dipartimento di Fisica, Politecnico di Milano, \\ Piazza L. Da Vinci 32, 20133 Milano, Italy
}

\begin{abstract}
We investigate, both numerically and experimentally, the optical parametric generation (OPG) process driven by femtosecond pulses in second-order nonlinear crystals. We discuss on the absolute phase and time fluctuations of the OPG beam. (C) 2010 Optical Society of America

OCIS codes: (190.4970) Parametric oscillators and amplifiers; (320.7110) Ultrafast nonlinear optics
\end{abstract}

Many studies of nonlinear light-matter interaction require ultrashort light pulses with broadly tunable carrier wavelength; in addition some applications such as time-resolved spectroscopy and nonlinear optics may require high pulse-to-pulse energy stability, accurate synchronization of two independent sources and control over the Carrier-Envelope phase (CEP) of the pulses. Optical parametric amplifiers (OPAs) are powerful instruments to address such requirements. The seed pulse of an OPA is usually produced by two means: optical parametric generation (OPG) [1] and white-light continuum (WLC) generation [2]: OPG is phase-matched parametric amplification of the vacuum field or quantum noise; WLC generation is a third-order nonlinear process where a combination of spatial self-focusing and temporal self-phase-modulation (SPM), lead to a dramatic spectral broadening. WLC presents high pulse-to-pulse stability and spatial beam quality and preserves the CEP of the driving pulse $[3,4]$, but requires driving pulses with energy higher than $1 \div 2 \mu \mathrm{J}$ and pulsewidth shorter than $200 \div 250$ fs. For those laser systems (such as Ytterbium-based bulk and fiber lasers) producing moderate energy pulses (a few $\mu \mathrm{Js}$ ) at high repetition rates (up to a few $\mathrm{MHz}$ ), the OPG approach is practically more attractive, especially because the availability of crystals with very high nonlinearity allows achieving large parametric gains with relatively low pump energies. Apart from conversion efficiency, other parameters should be considered to determine if OPG is a good source of femtosecond pulses, such as energy fluctuations, timing jitter and CEP of the generated pulses.

Since OPG is initiated by vacuum fluctuations or quantum noise, one would expect that the CEP link between the pump and the OPG pulses is weak or completely lost. However, in a recent paper [5] Hauri et al. reported CEP-stabilization of the idler of an OPG-seeded OPA, implying that the OPG process preserves the CEP of the pump pulse. This result calls for a comprehensive theoretical and experimental study of the CEPpreserving properties of the OPG process, which is currently lacking.
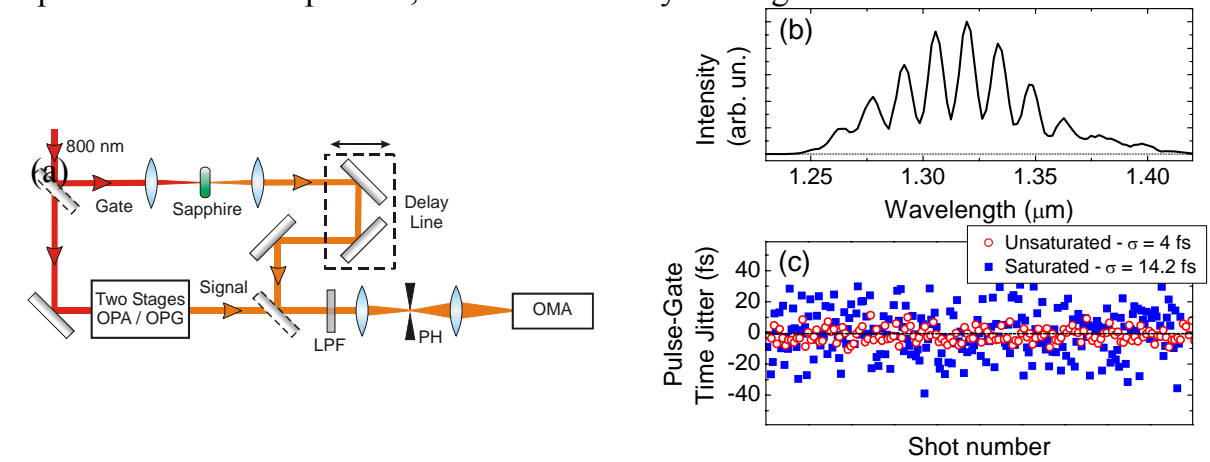

Fig. 1: (a) $f$-to- $f$ interferometer to measure the phase and time jitter between signal and gate pulses. PH: $50-\mu \mathrm{m}$ pin-hole. Pulses under investigation come either from an amplified WL beam or the OPG process at saturation; (b) single-shot interferometric pattern between Signal and Gate pulses; (c) Signal-Gate temporal jitter deduced from a sequence of interferograms recorded below and above saturation.

In this work we have investigated, both experimentally and numerically, the OPG process driven by femtosecond pulses, focusing on parameters such as CEP and time jitter. The OPG process is studied both below and above gain saturation.

For the experimental characterization of parametric generation, we used OPG crystals with different thicknesses: (i) the CEP properties of OPG below saturation were investigated with a 3-mm-thick BBO crystal; (ii) the time jitter of the OPG pulses in the saturation regime was characterized in 1-cm-thick BBO crystal. The 
system was powered by a regeneratively amplified Ti:sapphire laser, operating at $1 \mathrm{kHz}$ repetition rate and providing pulses with $1-\mathrm{mJ}$ energy, 50 -fs duration 800 -nm wavelength. The OPG crystals were cut at $\theta \approx 26^{\circ}$ for type II interaction, and generated an ordinary signal at $\sim 1.3 \mu \mathrm{m}$ and an extraordinary idler at $\sim 2.08 \mu \mathrm{m}$. Both CEP and time jitter of the OPG pulses were characterized by the $f$-to- $f$ interferometer shown in Fig. 1(a): one arm of the interferometer provided the gate pulse, which was a fraction of the 800-nm fundamental beam, spectrally broadened by WLC generation in a 3-mm-thick sapphire plate to allow spectral overlap with the signal pulse. The signals under investigation, arising from the OPG, were sent to the second arm of the interferometer, equipped with an optional two-stage OPA for amplification to the $\mu \mathrm{J}$-level of the low energy pulses from the unsaturated OPG. Signal and gate were then synchronized, projected to the same polarization and collinearly combined into an InGaAs spectrometer with single-shot detection capability at $1 \mathrm{kHz}$. The two beams give rise to interference fringe patterns [see Fig. 1(b)], which provides two different pieces of information: their period is related to the delay between signal and gate, whereas their phase is linked to the CEP phase shift of the signal with respect to the gate. The $f$-to- $f$ interferometer is therefore able to characterize, on a shot-to-shot basis, the fluctuations of time delay and CEP of the signal pulse with respect to the gate.

We obtained that the CEP of the OPG pulses exhibits pulse-to-pulse random fluctuations in the $(-\pi, \pi)$ range, thus confirming that the OPG process is not able to preserve the CEP of the pump pulses. Fig. 1(c) shows the OPG pulses time jitter, deduced from the fringe period: when OPG is driven below saturation (circles), the time jitter is of the order of $4 \mathrm{fs}$ confirming that signal is substantially temporally locked with the pump pulses; the same measurement in the saturation regime (squares) conveys that the jitter can be as high as $55 \mathrm{fs}$, comparable to the 50 -fs pump duration and larger than the expected OPG pulse duration of $\approx 36 \mathrm{fs}$.

The measured CEP and time jitter were in very good agreement with the results of numerical simulations: our model [6] described the OPG process by coupled nonlinear wave equations initiated by a noise field mimicking the quantum-mechanical source of superfluorescence. The simulations predicted that the Signal CEP is not correlated with the pump absolute phase; in addition they showed that the signal pulse is emitted with time fluctuations of the order of $\sigma=1 \mathrm{fs}$ and $14.5 \mathrm{fs}$ before and after gain saturation respectively, as also confirmed by the measurements. The role of saturation on the time jitter is related to the favorable group velocity mismatch values among pump signal and idler pulses in a type II interaction [7], and are clearly shown in the 2D maps reported in Fig. 2: here we represent the simulated temporal evolution of signal pulses during their interaction with the pump along the $1-\mathrm{cm}$ crystal, from the fluctuating random fields to the saturated propagation. The nonlinear interaction with the pump traps signal and idler pulses under the pump envelope, and the three pulses are nearly perfectly synchronized: when the OPG process reaches saturation, this nonlinear effect vanishes, and signal and idler pulses experience linear propagation, walking away from the pump with their own group velocity. Panels (a) and (b) compare situations initiated by two different noise fields: the growth of the signal pulses sets in at different crystal depths, thus influencing the temporal separation between pump and signal at the crystal output: saturation and different input noise fields thus concur to strong pulse-to-pulse fluctuations.

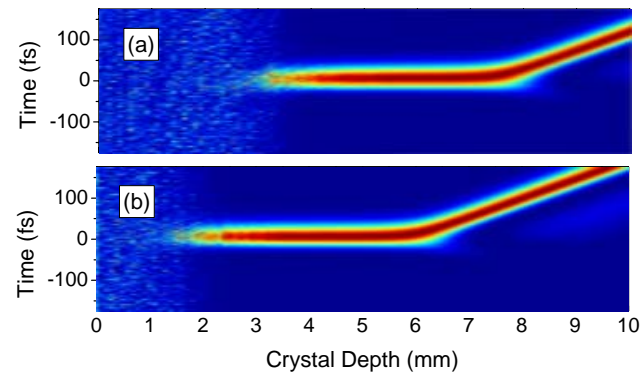

Fig. 2: Temporal evolution of intensity-normalized signal fields along the crystal, in a frame of reference moving with the pump; situations (a) and (b) differ only for the random noise fields initiating the OPG process.

In conclusion, we have investigated, both experimentally and numerically, the OPG process driven by femtosecond pulses, evaluating parameters such as CEP and time jitter. Both approaches demonstrated that there is no correlation between the CEPs of the OPG pulse and the pump pulse; in addition, when the OPG process is driven into saturation, the generated pulses exhibit a strong temporal jitter with respect to the driving pulses. These results indicate that OPG-seeded OPAs cannot be used in passive CEP stabilization schemes and applications in which absolute synchronization between two optical pulses is needed.

\section{References}

[1] M. Nisoli, S. Stagira, S. De Silvestri, O. Svelto, G. Valiulis and A. Varanavicius, Opt. Lett. 23, 630, 1998

[2] V. V. Yakovlev, B. Kohler, and K. R. Wilson, Opt. Lett. 19, 2000 (1994).

[3] M. Bellini and T. W. Hänsch, Opt. Lett. 25, 1049 (2000).

[4] P. Baum, E. Riedle, M. Greve, and H. R. Telle, Opt. Lett. 30, 2028 (2005).

[5] C. P. Hauri, R. B. Lopez-Martens, C. I. Blaga, K. D. Schultz, J. Cryan, R. Chirla, P. Colosimo, G. Doumy, A. M. March, C. Roedig, E. Sistrunk, J. Tate, J. Wheeler, L. F. DiMauro, and E. P. Power, Opt. Lett. 32, 868 (2007).

[6] C. Manzoni , G. Cirmi, D. Brida, S. De Silvestri and G. Cerullo, Phys. Rev. A 79, 033818 (2009)

[7] R. Danielius, A Piskarskas, A. Stabinis, G. P. Banfi, P. Di Trapani, and R. Righini, J. Opt. Soc. Am. B 10, 2222 (1993). 